\title{
A Performance Comparison of FBMC Modulation Schemes with Short Perfect Reconstruction Filters
}

\author{
Damien Roque and Cyrille Siclet \\ Grenoble Images Parole Signal Automatique (GIPSA-lab) \\ 11, rue des Mathématiques \\ 38402 Grenoble, FRANCE \\ Email: \{damien.roque,cyrille.siclet\}@ gipsa-lab.grenoble-inp.fr
}

\author{
Pierre Siohan \\ Orange-Labs \\ 4, rue du Clos Courtel \\ 35512 Cesson Sévigné, FRANCE \\ Email: pierre.siohan@orange.com
}

\begin{abstract}
In this paper, we study the performance of filter bank based multicarrier (FBMC) transmission systems over doubly-dispersive channels. FBMC generalizes traditional orthogonal frequency-division multiplexing (OFDM) schemes, allowing a non-rectangular sub-channel pulse shape in the time domain. This approach leads to a better spectral containment that improves interference mitigation in several time-variant environments. A general analysis describes the transmultiplexer structure and the time-variant multipath channel model. We specify rectangular filters and short perfect reconstruction (PR) filters that give rise to two families of FBMC transceivers. A performance comparison between these schemes is performed in terms of bit-error-rate using a single-tap per sub-channel equalizer. This study emphasizes the low complexity of the FBMC transceivers in the case of short filters and discusses its usefulness through simulation results in two mobility scenarios.
\end{abstract}

\section{INTRODUCTION}

Cyclic-prefix orthogonal frequency-division multiplexing (CP-OFDM) diagonalizes time-invariant multipath channels provided a guard interval longer than the channel impulse response. Perfect reconstruction may be obtained with onetap equalization per sub-channel [1]. Furthermore, an efficient implementation using fast Fourier transform (FFT) makes CPOFDM an attractive modulation scheme, widely used for both wired and wireless communications (e.g. IEEE 802.11a, DVB$\mathrm{T}$, xDSL).

However, when transmitting a CP-OFDM signal over a time-variant multipath channel, the Doppler spread induces inter-carrier interference (ICI) and increases error probability. The lack of frequency localization of the rectangular waveform is no longer compensated by the cyclic prefix in presence of Doppler spread [2, p. 753].

A more general transmission framework is carried out by filter bank based multicarrier modulations (FBMC), also known as filtered multitone (FMT) or oversampled OFDM [3], [4]. The key idea behind this technique is to perform a nonrectangular pulse-shaping as compliant as possible with the channel characteristics (time and frequency dispersion). The design of pulse-shaping filters is an active area of research [5]-[9].

In this work, we focus on non-rectangular (NR) perfect reconstruction short filters, introduced in [10]. The subsequent multicarrier transmission system is referred to as NR-OFDM.
In opposition to arbitrary long pulses, the extra implementation cost compared to CP-OFDM becomes negligible with this particular class of prototype filters [11]. Furthermore, the design method leads to closed-form expressions.

Our contributions include bit-error-rate comparison of $\mathrm{CP}$ OFDM and NR-OFDM over a realistic time-frequency dispersive channel, extending the work developed in [12] over NR short filters (through simulation results).

This paper is organized as follows. Section 2 presents the general transmultiplexer structure and the channel model used for performance measurement. Section 3 focuses on the mean signal-to-noise-plus-interference ratio (SINR). Section 4 defines rectangular and perfect reconstruction filters. Section 5 compares the performances of CP-OFDM and NR-OFDM through simulation results. Finally, conclusions and perspectives are presented in section 6 .

\section{SYSTEM MODEL}

We briefly introduce a generalized multicarrier transmission system through a discrete-time formulation, in order to underline digital implementation.

\section{A. FBMC Transmitter}

Let $\left\{c_{m, n}\right\}_{(m, n) \in \Lambda}$ denote a complex symbol sequence where $\boldsymbol{\Lambda}=\{0, \ldots, M-1\} \times \mathbf{Z}$. We assume independent and identically distributed symbols. Their average power is given by $\sigma_{c}^{2}=\mathrm{E}\left\{\left|c_{m, n}\right|^{2}\right\}$.

Each $c_{m, n}$ is distributed in the time-frequency plane at coordinates $(n N, m / M)$ where $N$ is the number of samples per sub-channel symbol period and $M$ represents the number of sub-channels. We call $N / M$ the oversampling ratio. The output of the discrete-time transmitter writes

$$
s[k]=\sum_{(m, n) \in \mathbf{\Lambda}} c_{m, n} \gamma_{m, n}[k], \quad k \in \mathbf{Z}
$$

where $\gamma_{m, n}[k]$ is a time-frequency shifted prototype pulse defined as

$$
\gamma_{m, n}[k]=\gamma[k-n N] e^{j 2 \pi \frac{m}{M} k}, \quad \gamma[k] \in \mathbf{R} .
$$

A necessary condition for perfect reconstruction of the transmitted symbols is that the functions $\left\{\gamma_{m, n}\right\}_{(m, n) \in \Lambda}$ are linearly independent. This implies $N / M \geq 1$, as proven by the 


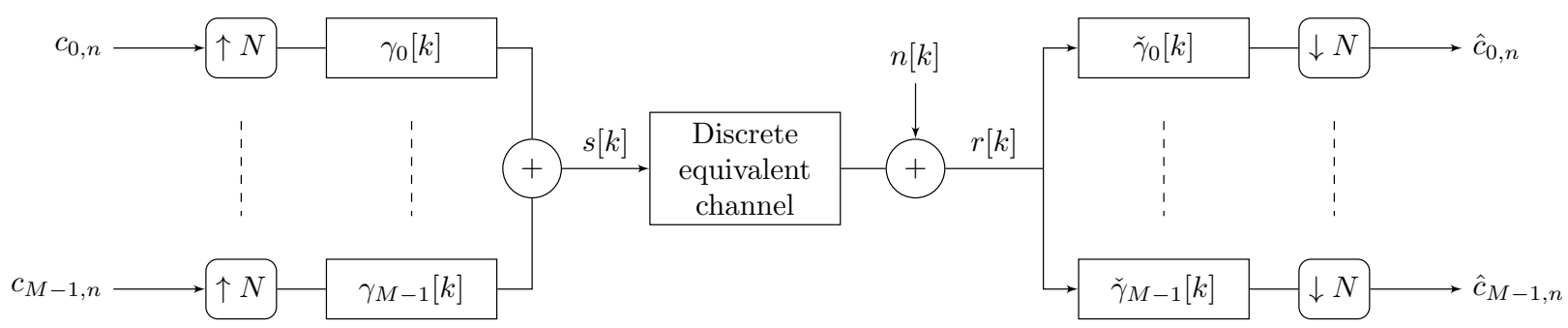

Fig. 1: Discrete-time representation of a FBMC transmission system with $\gamma_{m}[k]=\gamma[k] \exp (j 2 \pi m k / M)$ and $\check{\gamma}_{m}[k]=\check{\gamma}[k] \exp (-j 2 \pi m k / M)$.

Weyl-Heisenberg (or Gabor) frame theory [13]. We note that orthogonality and completeness are not mandatory conditions for perfect reconstruction [3].

\section{B. Channel model}

Let us assume a bandlimited version of $s[k]$, the WhittakerShannon interpolation implies

$$
s(t)=\sum_{k \in \mathbf{Z}} s[k] \operatorname{sinc}(B t-k), \quad t \in \mathbf{R}
$$

where $\operatorname{sinc}(x)=\sin (\pi x) / \pi x$ and $B$ is the overall transmission bandwidth.

In a mobile radio context, we consider a time-frequency dispersive channel $\mathcal{H}$, with $I$ resolvable paths. Assuming a baseband equivalent model, the continuous-time input-output relation is given by

$$
\begin{aligned}
r(t) & =(\mathcal{H} s)(t)+n(t) \\
& =\sum_{i=1}^{I} \alpha_{i}(t) s\left(t-\tau_{i}\right)+n(t)
\end{aligned}
$$

where $\alpha_{i}(t)$ is a complex gain associated to the $i$ th path with delay $\tau_{i}$. The complex bandlimited Gaussian noise $n(t)$ is characterized by its power spectral density equal to $\sigma_{n}^{2} / B$ for $|f| \leq B / 2$ and 0 otherwise:

$$
n(t)=\sum_{k \in \mathbf{Z}} n[k] \operatorname{sinc}(B t-k), \quad t \in \mathbf{R} .
$$

A statistical channel model is introduced in [14] and relies on wide sense stationarity and uncorrelated scatterers assumptions (WSSUS), this implies

$$
\mathrm{E}\left\{\alpha_{i}^{*}(t) \alpha_{i^{\prime}}\left(t^{\prime}\right)\right\}=\Gamma_{i}\left(t-t^{\prime}\right) \delta_{i, i^{\prime}}
$$

where $\Gamma_{i}$ represents the autocorrelation function of the $i$ th group of scatterers and $\delta_{i, i^{\prime}}$ is the Kronecker delta. For example, using Clarke's isotropic dense scattering model [15], $\alpha_{i}(t)$ are complex Gaussian processes with

$$
\Gamma_{i}\left(t-t^{\prime}\right)=J_{0}\left(2 \pi f_{d}\left(t-t^{\prime}\right)\right)
$$

where $J_{0}$ denotes the zeroth order Bessel function of the first kind and $f_{d}$ represents the maximum Doppler shift. The well known Jakes Doppler spectrum is obtained by a Fourier transform of (8) and is equal to

$$
C_{i}(f)= \begin{cases}\frac{1}{\pi f_{d} \sqrt{1-\left(f / f_{d}\right)^{2}}}, & \text { if }|f|<f_{d} \\ 0, & \text { otherwise. }\end{cases}
$$

For simulation purpose, we derive a discrete-time version of the channel model denoted $\tilde{\mathcal{H}}$. Using (3) and (4), the inputoutput relation becomes

$$
\begin{aligned}
r[k] & =(\tilde{\mathcal{H}} s)[k]+n[k] \\
& =\sum_{l \in \mathbf{Z}} \tilde{\alpha}_{l}[k] s[k-l]+n[k]
\end{aligned}
$$

with

$$
\tilde{\alpha}_{l}[k]=\sum_{i=1}^{I} \alpha_{i}\left(\frac{k}{B}\right) \operatorname{sinc}\left(B \tau_{i}-l\right) .
$$

We notice that correlation among the $1 / B$-spaced channel taps is introduced by anti-aliasing filtering so that

$$
\mathrm{E}\left\{\tilde{\alpha}_{l}^{*}[k] \tilde{\alpha}_{l^{\prime}}\left[k^{\prime}\right]\right\}=\Omega_{l, l^{\prime}}\left[k-k^{\prime}\right]
$$

where

$\Omega_{l, l^{\prime}}\left[k-k^{\prime}\right]=\sum_{i=1}^{I} \operatorname{sinc}\left(B \tau_{i}-l\right) \operatorname{sinc}\left(B \tau_{i}-l^{\prime}\right) \Gamma_{i}\left(\frac{k-k^{\prime}}{B}\right)$.

Moreover, the band limiting operation leads to an infinite number of virtual coefficients $\left\{\tilde{\alpha}_{l}[k]\right\}_{k \in \mathbf{Z}}$. In practice, this sequence is truncated whenever $\left|\tilde{\alpha}_{l}[k]\right|^{2}$ becomes negligible. The resulting error is discussed in [16].

\section{FBMC Receiver}

Considering $x$ and $y$ two functions of $\ell_{2}(\mathbf{Z})$, we define the inner product $\langle x, y\rangle=\sum_{k \in \mathbf{Z}} x^{*}[k] y[k]$ and its induced norm $\|x\|=\sqrt{\langle x, x\rangle}$. Then, the dual frame of $\left\{\gamma_{m, n}\right\}_{(m, n) \in \mathbf{\Lambda}}$ is

$$
\check{\gamma}_{m, n}[k]=\check{\gamma}[k-n N] e^{j 2 \pi \frac{m}{M} k}, \quad \check{\gamma}[k] \in \mathbf{R} .
$$

The biorthogonality condition is given by $\left\langle\check{\gamma}_{p, q}, \gamma_{m, n}\right\rangle=$ $\delta_{m, p} \delta_{n, q}$ for all $(m, n)$ and $(p, q)$ in $\boldsymbol{\Lambda}$. The transmitted symbols are recovered by computing the inner product of the received signal and the dual frame $\left\{\check{\gamma}_{m, n}\right\}_{(m, n) \in \Lambda}$ (Fig. 1). We denote $\boldsymbol{\Lambda}_{\mathbf{p}, \mathbf{q}}=\boldsymbol{\Lambda} \backslash\{(p, q)\}$ and we express the $q$ th received symbol on the $p$ th sub-channel using (1) and (10):

$$
\begin{aligned}
\hat{c}_{p, q} & =\left\langle\check{\gamma}_{p, q}, r\right\rangle \\
& =\left\langle\check{\gamma}_{p, q}, \tilde{\mathcal{H}} s\right\rangle+\left\langle\check{\gamma}_{p, q}, n\right\rangle \\
& =\underbrace{c_{p, q} A_{p, q}}_{S_{p, q}}+\underbrace{\sum_{(m, n) \in \Lambda_{\mathbf{p}, \mathbf{q}}} c_{m, n} A_{m, n, p, q}}_{I_{p, q}}+N_{p, q}
\end{aligned}
$$


where

$$
\begin{aligned}
A_{m, n, p, q}= & \left\langle\check{\gamma}_{p, q}, \tilde{\mathcal{H}} \gamma_{m, n}\right\rangle \\
= & \sum_{k \in \mathbf{Z}} \check{\gamma}[k-q N] e^{j 2 \pi \frac{(m-p)}{M} k} \\
& \cdot \sum_{l \in \mathbf{Z}} \tilde{\alpha}_{l}[k] \gamma[k-l-n N] e^{-j 2 \pi \frac{m}{M} l} \\
A_{p, q}= & \left\langle\check{\gamma}_{p, q}, \tilde{\mathcal{H}} \gamma_{p, q}\right\rangle=A_{p, q, p, q}
\end{aligned}
$$

and

$$
N_{p, q}=\left\langle\check{\gamma}_{p, q}, n\right\rangle=\sum_{k \in \mathbf{Z}} n[k] \check{\gamma}[k-q N] e^{-j 2 \pi \frac{p}{M} k} .
$$

The expression of the received symbol splits into three terms:

- $S_{p, q}$ represents the useful part of the signal;

- $I_{p, q}$ denotes the interference term, including inter-carrier and inter-symbol interference;

- $N_{p, q}$ refers to the noise filtered by the receiver.

The coefficient $A_{m, n, p, q}$ reflects the complex gain introduced by the system, assuming an estimated symbol $(p, q)$ and an interfering symbol $(m, n)$.

In order to maintain a low-complexity receiver, interference mitigation is performed by a zero-forcing equalizer, using one coefficient per sub-channel. Assuming perfect knowledge of $\left\{\tilde{\alpha}_{l}[k]\right\}(l, k) \in \mathbf{Z}^{2}$, the expression of the equalized signal is given by

$$
\tilde{c}_{p, q}=c_{p, q}+\frac{I_{p, q}}{A_{p, q}}+\frac{N_{p, q}}{A_{p, q}} .
$$

This type of equalizer presents the advantage of a low complexity but it may amplify noise and interference terms when the channel experience severe fading [1].

\section{SINR MAXIMIZATION IN THE WSSUS CONTEXT}

The design of a multicarrier transmission system consists in optimizing the parameters $M, N, \gamma, \check{\gamma}$ subject to a given channel realization. Such a joint optimization problem remains unsolved since there is an arbitrary trade-off between interference level and spectral efficiency [8]. Most studies in this field choose a separate optimization approach on $S_{p, q}, I_{p, q}$ and $N_{p, q}$ for a given lattice structure (i.e. $M$ and $N$ fixed).

Furthermore, the channel is often considered as time-variant over a block. Thus, an optimization based on the second-order statistics is usually performed [3], [8].

In the following optimization procedure, we consider a normalized transmission pulse shape, such that $\|\gamma\|^{2}=1$. We denote $\sigma_{S}^{2}=\mathrm{E}_{c, \tilde{\mathcal{H}}}\left\{\left|S_{p, q}\right|^{2}\right\}, \sigma_{I}^{2}=\mathrm{E}_{c, \tilde{\mathcal{H}}}\left\{\left|I_{p, q}\right|^{2}\right\}$ and $\sigma_{N}^{2}=\mathrm{E}_{n}\left\{\left|N_{p, q}\right|^{2}\right\}$. We conduct here a qualitative analysis of the signal-to-noise ratio $\left(\mathrm{SNR}=\sigma_{S}^{2} / \sigma_{N}^{2}\right)$ and signal-tointerference ratio $\left(\mathrm{SIR}=\sigma_{S}^{2} / \sigma_{I}^{2}\right)$. A more thorough study is developed in [6], [17]. The expression of the signal-to-noiseplus-interference ratio is then given by

$$
\mathrm{SINR}=\frac{\sigma_{S}^{2}}{\sigma_{N}^{2}+\sigma_{I}^{2}}=\frac{1}{\frac{1}{\mathrm{SNR}}+\frac{1}{\mathrm{SIR}}} .
$$

\section{A. SNR maximization}

Assuming an additive complex bandlimited Gaussian noise channel such that $n[k] \sim \mathcal{C N}\left(0, \sigma_{n}^{2}\right)$, the mean power of the received noise term $N_{p, q}$ writes

$$
\mathrm{SNR}=\frac{\sigma_{S}^{2}}{\sigma_{N}^{2}}=\frac{\sigma_{S}^{2}}{\sigma_{n}^{2}\|\check{\gamma}\|^{2}}
$$

The biorthogonality condition applied to the prototype function implies $\langle\gamma, \check{\gamma}\rangle=1$. Using the Cauchy-Schwarz inequality we also have $|\langle\gamma, \check{\gamma}\rangle| \leq\|\gamma\|^{2}\|\check{\gamma}\|^{2}$ and finally

$$
\|\gamma\|^{2}\|\check{\gamma}\|^{2} \geq 1 \text {. }
$$

The average noise power is minimum when equality is verified in (26). It requires an orthogonal setup, that is to say $\check{\gamma}=\beta \gamma$, $\forall \beta \in \mathbf{R}$. This result corroborates the matched filter theory [2, p. 160].

\section{B. SIR maximization}

Independence between symbols and channel coefficients allows us to write

$$
\mathrm{SIR}=\frac{\sigma_{S}^{2}}{\sigma_{I}^{2}}=\frac{\mathrm{E}_{\tilde{\mathcal{H}}}\left\{\left|A_{p, q}\right|^{2}\right\}}{\sum_{(m, n) \in \boldsymbol{\Lambda}_{\mathbf{p}, \mathbf{q}}} \mathrm{E}_{\tilde{\mathcal{H}}}\left\{\left|A_{m, n, p, q}\right|^{2}\right\}}
$$

where

$$
\begin{aligned}
\mathrm{E}_{\tilde{\mathcal{H}}}\{\mid & \left.\left.A_{m, n, p, q}\right|^{2}\right\} \\
= & \sum_{k, k^{\prime}} \sum_{l, l^{\prime}} \mathrm{E}\left\{\tilde{\alpha}_{l}^{*}[k+q N] \tilde{\alpha}_{l^{\prime}}\left[k^{\prime}+q N\right]\right\} \\
& \cdot \check{\gamma}[k] \check{\gamma}\left[k^{\prime}\right] \gamma[k-l-(n-q) N] \gamma\left[k^{\prime}-l^{\prime}-(n-q) N\right] \\
& \cdot e^{j 2 \pi \frac{(m-p)}{M}\left(k-k^{\prime}\right)} e^{-j 2 \pi \frac{m}{M}\left(l-l^{\prime}\right)} .
\end{aligned}
$$

In light of (27), the signal-to-interference ratio may be maximized if $\mathrm{E}_{\tilde{\mathcal{H}}}\left\{\left|A_{m, n, p, q}\right|^{2}\right\} \rightarrow \delta_{m, p} \delta_{n, q}$ subject to given values of $M, N$ and the second-order characteristics of the WSSUS channel.

We assume $\Omega_{l, l^{\prime}}\left[k-k^{\prime}\right] \approx \sigma_{l}^{2}\left[k-k^{\prime}\right] \delta_{l, l^{\prime}}$, where $\sigma_{l}^{2}\left[k-k^{\prime}\right]=$ $\sum_{i=1}^{I} \operatorname{sinc}^{2}\left(B \tau_{i}-l\right) \Gamma_{i}\left[k-k^{\prime}\right]$. This is a convenient approximation when the sampling period $1 / B$ is small compared to time arrivals $\tau_{i}$. Considering the same Doppler spectrum for each path, we have

$$
\mathrm{E}_{\tilde{\mathcal{H}}}\left\{\left|A_{m, n, p, q}\right|^{2}\right\}=\sum_{k, k^{\prime}} Q_{n-q}\left[k, k^{\prime}\right] e^{j 2 \pi \frac{(m-p)}{M}\left(k-k^{\prime}\right)}
$$

where

$$
Q_{n-q}\left[k, k^{\prime}\right]=\Gamma\left[k-k^{\prime}\right] \check{\gamma}\left[k^{\prime}\right] \check{\gamma}[k] R_{n-q}\left[k, k^{\prime}\right]
$$

and

$$
\begin{aligned}
R_{n-q}\left[k, k^{\prime}\right]= & \sum_{l \in \mathbf{Z}} \sigma_{l}^{2}\left[k-k^{\prime}\right] \gamma[k-l-(n-q) N] \\
& \cdot \gamma\left[k^{\prime}-l-(n-q) N\right] .
\end{aligned}
$$

We notice that $R_{n-q}\left[k, k^{\prime}\right]$ reflects the time-autocorrelation of the transmitter pulse shape. On one hand, we want $R_{n-q}\left[k, k^{\prime}\right] \approx \delta_{n, q}$. On the other hand, (29) requires $Q_{n-q}\left[k, k^{\prime}\right]$ to be approximately constant in order to satisfy 
$\mathrm{E}_{\tilde{\mathcal{H}}}\left\{\left|A_{m, n, p, q}\right|^{2}\right\} \approx \delta_{m, p}$. These two constraints are incompatible and demonstrate the time-frequency uncertainty principle. An optimal trade-off may be reached using Gaussian pulses. However, due to their non-orthogonality, one may need to choose a large oversampling factor in order to mitigate ISI and ICI. Another frequently used strategy is to perform an orthogonalization procedure, as described in [8].

\section{SINR maximization}

Summarizing the SNR and SIR maximization constraints, a quasi-optimal transceiver may be designed in terms of mean SINR, for a given $M, N$ and $\Gamma[k]$, if the following conditions hold:

- orthogonal scheme such that $\check{\gamma}=\beta \gamma, \beta \in \mathbf{R}$;

- good time-frequency localization for $\gamma$ and $\check{\gamma}$;

- high spectral efficiency such that $N / M \rightarrow 1$.

However, the last two conditions cannot be satisfied at the same time [18, p. 63]. If we relax the spectral efficiency condition, it becomes possible to decrease interference mean power. This is of primary concern when experiencing fastfading.

\section{FILTERS SPECIFICATION}

In this section, we study two families of prototype filters that are used for multicarrier communications over timefrequency dispersive channels: rectangular and short perfect reconstruction filters.

From the optimization guidelines described above, it is of prime interest to characterize the filters in the time-frequency plane. We recall the second-order moments in time and frequency assuming a real-valued linear-phase discrete-time signal $x[k] \in \ell_{2}(\mathbf{Z})$ of length $L$ :

$$
\begin{aligned}
& \sigma_{t}^{2}(x)=\frac{1}{\|x\|^{2}} \sum_{k=0}^{L-1}\left(k-\frac{L-1}{2}\right)^{2}|x[k]|^{2}, \\
& \sigma_{f}^{2}(x)=\frac{1}{\|x\|^{2}} \int_{-\frac{1}{2}}^{\frac{1}{2}} f^{2}|X(f)|^{2} \mathrm{~d} f
\end{aligned}
$$

with $X(f)=\sum_{k=0}^{L-1} x[k] \exp (-j 2 \pi f k / L)$. The out-of-band energy with respect to a band $1 / M$ writes

$$
\epsilon_{M}(x)=\frac{1}{\|x\|^{2}} \int_{\frac{1}{2 M}}^{\frac{1}{2}}|X(f)|^{2} \mathrm{~d} f .
$$

The time-frequency coefficient is given by

$$
\xi(x)=\frac{1}{4 \pi \sigma_{t}(x) \sigma_{f}(x)} .
$$

If $X( \pm 1 / 2)=0$ then, according to [19], $0 \leq \xi(x)<1$.

\section{A. Rectangular filters (CP-OFDM)}

CP-OFDM relies on a biorthogonal FBMC scheme based on rectangular filters defined by

$$
\begin{aligned}
\gamma_{\mathrm{RECT}}[k] & =\frac{1}{\sqrt{N}} \chi_{\{0, \ldots, N-1\}}[k] \\
\check{\gamma}_{\mathrm{RECT}}[k] & =\frac{\sqrt{N}}{M} \chi_{\{N-M, \ldots, N-1\}}[k]
\end{aligned}
$$

where $\chi_{\mathbf{I}}$ is the indicator function of any set $\mathbf{I}$, specified by

$$
\chi_{\mathbf{I}}[k]= \begin{cases}0, & \text { if } k \notin \mathbf{I} \\ 1, & \text { if } k \in \mathbf{I} .\end{cases}
$$

The first $N-M$ coefficients of $\gamma$ form the cyclic prefix in order to mitigates ISI introduced by time-dispersive channels. At the receiver side the cyclic-prefix is removed by $\check{\gamma}$. It follows from (25) that a biorthogonal setup amplifies the noise term since $\|\check{\gamma}\|^{2}>1$.

\section{B. Short perfect reconstruction filters (NR-OFDM)}

NR-OFDM refers to an orthogonal FBMC scheme using perfect reconstruction filters (i.e. in addition to the biorthogonality condition, we have $\gamma=\check{\gamma}$ ). In this article, we focus on short filters so that their length is equal to $N$. This class of filters allows an efficient implementation which is comparable with CP-OFDM. The pulse-shaping procedure requires $N$ additional multiplications at the transmitter and at the receiver side [11].

The prototypes described in [10] are derived from two optimization criteria: out-of-band energy minimization (OBE) and time-frequency localization maximization (TFL). Using $\Delta=N-M$, closed-form expressions are given for both filters.

$\gamma_{\mathrm{OBE}}[k]= \begin{cases}\frac{1}{\sqrt{M}} \cos \left(\tilde{a}+\tilde{b} \frac{(2 k+1)}{2 \Delta}\right) & \text { if } 0 \leq k \leq \Delta-1 \\ \frac{1}{\sqrt{M}} & \text { if } \Delta \leq k \leq M-1 \\ \frac{1}{\sqrt{M}} \cos \left(\tilde{a}+\tilde{b} \frac{2(\Delta-k)+1}{2 \Delta}\right) & \text { if } M \leq k \leq N-1\end{cases}$

$$
\gamma_{\mathrm{TFL}}[k]= \begin{cases}\frac{1}{\sqrt{M}} \sin \left(\frac{(2 k+1) \pi}{4 \Delta}\right) & \text { if } 0 \leq k \leq \Delta-1 \\ \frac{1}{\sqrt{M}} & \text { if } \Delta \leq k \leq M-1 \\ \frac{1}{\sqrt{M}} \sin \left(\frac{(2(\Delta-k)+1) \pi}{4 \Delta}\right) & \text { if } M \leq k \leq N-1\end{cases}
$$

where $\tilde{a}$ and $\tilde{b}$ are two constants given in [10] and depending on the ratio $M / \Delta$.

A time-frequency analysis of the prototypes is summarized by Fig. 2 and Tab. I. Considering the empirical computation of time-frequency moments, a rectangular pulse is submitted as a reference. We show that TFL pulses demonstrate a better localization both in time and in frequency than OBE. As expected, the time-frequency localization of the rectangular waveform is far behind OBE and TFL pulses.

TABLE I: Filters characteristics in the time-frequency domain for $M=64$ and $N=72$.

\begin{tabular}{|c|c|c|c|c|}
\hline Filter type & $\sigma_{t}(\check{\gamma})$ & $\sigma_{f}(\check{\gamma})$ & $\epsilon_{M}(\check{\gamma})$ & $\xi(\check{\gamma})$ \\
\hline RECT & $0.2566 N$ & $2.12 / M$ & 0.2263 & 0.1226 \\
\hline NR-OBE & $0.2617 N$ & $1.44 / M$ & 0.1715 & 0.1874 \\
\hline NR-TFL & $0.2580 N$ & $0.68 / M$ & 0.1839 & 0.4047 \\
\hline
\end{tabular}



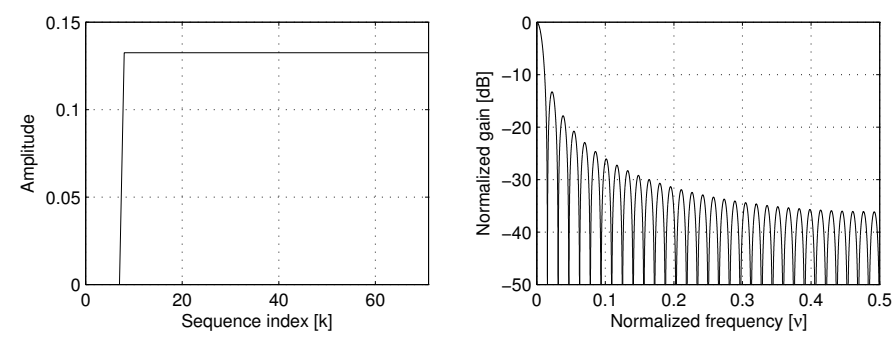

(a) Rectangular filter $\left(\check{\gamma}_{\text {RECT }}\right)$.
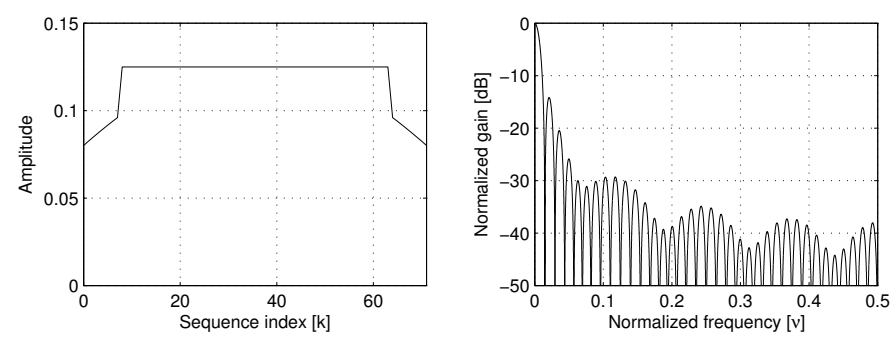

(b) Perfect reconstruction OBE filter.
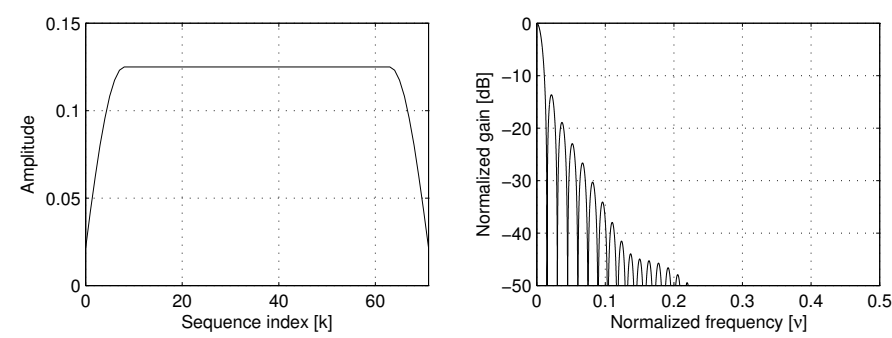

(c) Perfect reconstruction TFL filter.

Fig. 2: Time and frequency responses of the prototypes filters for $M=64$ and $N=72$.

\section{Simulations Results}

The scenario developed in our simulations refers to a video broadcasting system operating around a central frequency $f_{c}=$ $600 \mathrm{MHz}$ and using a band $B=8 \mathrm{MHz}$. We consider here two mobility scenarios: pedestrian $\left(v_{\max }=3 \mathrm{~km} / \mathrm{h}\right)$ and vehicular $\left(v_{\max }=350 \mathrm{~km} / \mathrm{h}\right)$. The propagation takes place in an urban environment, this justifies the use of COST 207 TUx6 channel model [20]. This involves a 6-path WSSUS model where the last path occurs at $5 \mu \mathrm{s}$.

The video stream is split into packets, transmitted in an asynchronous way (i.e. different packets experience independent realizations of the channel). A packet is made of several OFDM symbols which includes $M=4096$ QPSK symbols. Simulations are computed for CP-OFDM, OBE-OFDM and TFL-OFDM with $N / M \in\{17 / 16,9 / 8,5 / 4\}$. Uncoded biterror-rate (BER) performances are presented as a function of $E_{b} / N_{0}$ where $E_{b}=N \sigma_{c}^{2} / 2 M B$ and $N_{0}=\sigma_{n}^{2} / B$ (Fig. 3).

First of all, the oversampling factor demonstrates a different behavior in the CP-OFDM case and in the NR-OFDM case. In fact, the length of the cyclic prefix has no consequence on the time-frequency localization of the rectangular pulse $\check{\gamma}$. However, according to (26) and (36), an increased $N / M$ factor amplifies the noise term. On the contrary NR filters show a better time-frequency localization when $N / M$ increases and improves the resilience to ISI and ICI.

In the low mobility scenario, the use of TFL-OFDM might be interesting because it provides a good frequency localization which is required for mainly frequency-selective channels (Fig. 3b). We notice that CP-OFDM remains an attractive solution for low $N / M$ factor.

On the contrary, the high mobility scenario combined with a narrow frequency spacing between subcarriers $(M=4096)$, first demonstrates the superiority of the NR-OFDM solutions compared to CP-OFDM. We also note that OBE-OFDM leads to a better result than TFL-OFDM. Indeed, compared to TFL, this criterion provides pulses with narrower main lobes in the frequency domain. This spectral characteristic tends to mitigate ICI introduced by Doppler spread (Fig. 3c).

Summarizing the simulation results, and some other ones not presented here, it appears that considering both mobility scenarios (low and high speed) the TFL prototype offers a good trade-off and, in presence of Doppler spread, always outperforms CP-OFDM for $M \in\{1024,2048,4096\}$ and $E_{b} / N_{0}<20 \mathrm{~dB}$.

\section{CONCLUSION}

We have presented an analysis of FBMC schemes in the context of time-frequency dispersive channels. Using WSSUS assumption, we have shown the interest of well localized pulses in the time-frequency domain in order to mitigate ISI and ICI. Focusing on the particular case of non-rectangular short perfect reconstruction filters [10], we preserve an acceptable transceiver complexity. Furthermore, we demonstrate through numerical simulations that one-tap equalization is sufficient to yield superior BER performance than CP-OFDM, for similar oversampling factors in several high mobility scenarios.

\section{ACKNOWLEDGMENT}

This work has been supported by the French defence procurement agency (DGA) and Orange-Labs. The authors would like to thank D. Pinchon who contributed to the design of the filters discussed in this article.

\section{REFERENCES}

[1] Z. Wang and G. B. Giannakis, "Wireless multicarrier communications," IEEE Signal Process. Mag., vol. 17, no. 3, pp. 29-48, 2000.

[2] J. Proakis and M. Salehi, Digital communications, 5th ed. McGrawHill, 2006.

[3] W. Kozek and A. F. Molisch, "Nonorthogonal pulseshapes for multicarrier communications in doubly dispersive channels," IEEE J. Sel. Areas Commun., vol. 16, no. 8, pp. 1579-1589, 1998.

[4] G. Cherubini, E. Eleftheriou, and S. Olcer, "Filtered multitone modulation for very high-speed digital subscriber lines," IEEE J. Sel. Areas Commun., vol. 20, no. 5, pp. 1016-1028, 2002.

[5] A. Vahlin and N. Holte, "Optimal finite duration pulses for OFDM," IEEE Trans. Commun., vol. 44, no. 1, pp. 10-14, 1996.

[6] T. Strohmer and S. Beaver, "Optimal OFDM design for time-frequency dispersive channels," IEEE Trans. Commun., vol. 51, no. 7, pp. 11111122, 2003.

[7] C. Siclet, P. Siohan, and D. Pinchon, "Perfect reconstruction conditions and design of oversampled DFT-modulated transmultiplexers," EURASIP journal on applied signal processing, vol. 2006, pp. 1-14, 2006. 


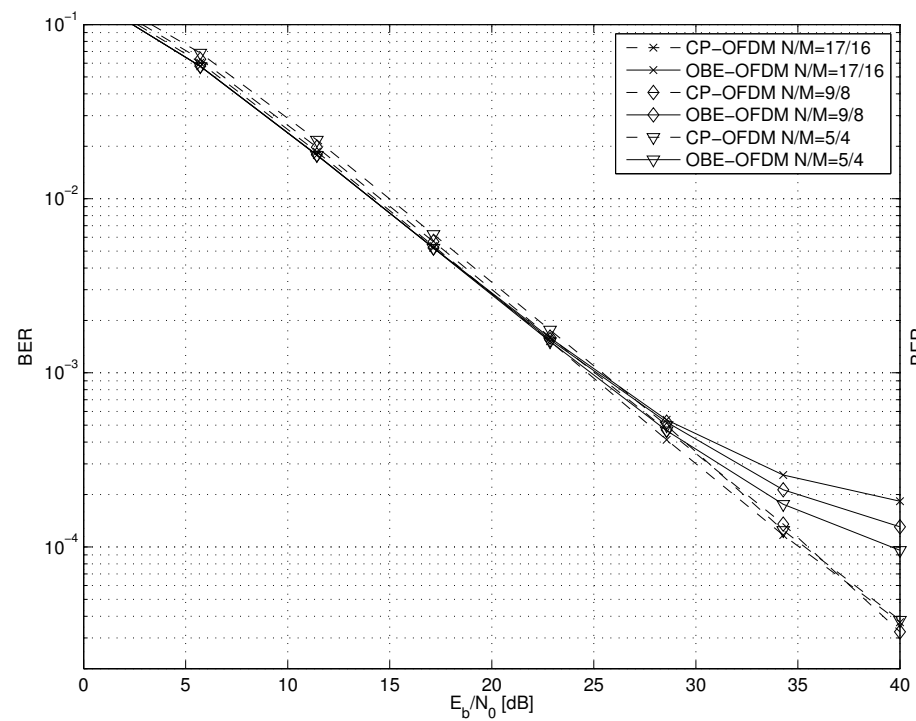

(a) BER performances of OBE-OFDM and CP-OFDM $\left(v_{\max }=3 \mathrm{~km} / \mathrm{h}\right)$.

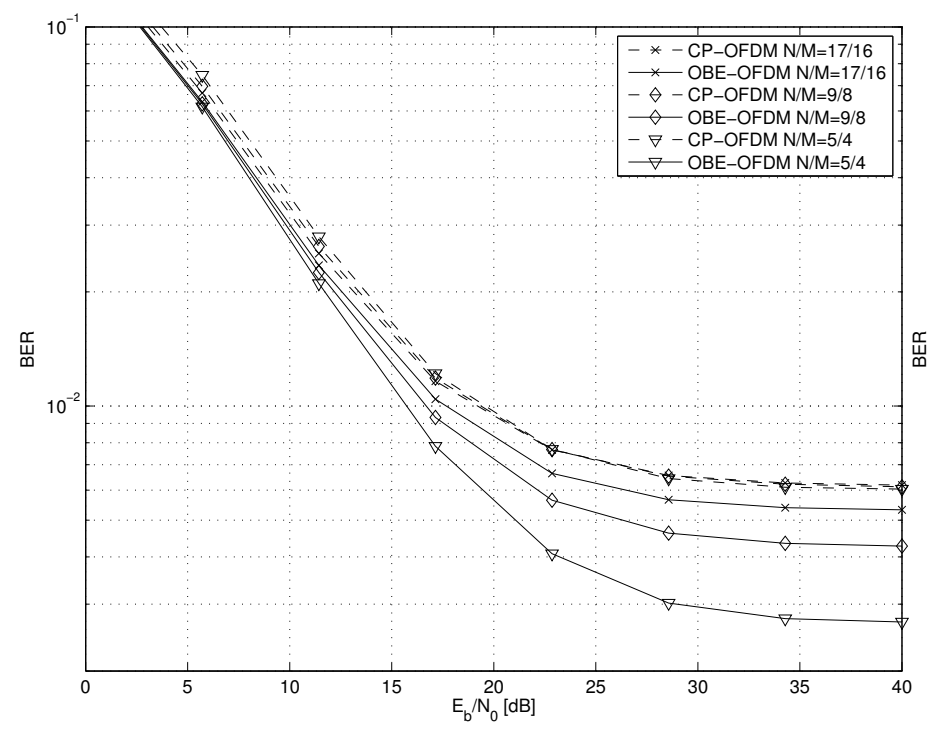

(c) BER performances of OBE-OFDM and CP-OFDM $\left(v_{\max }=350 \mathrm{~km} / \mathrm{h}\right)$.

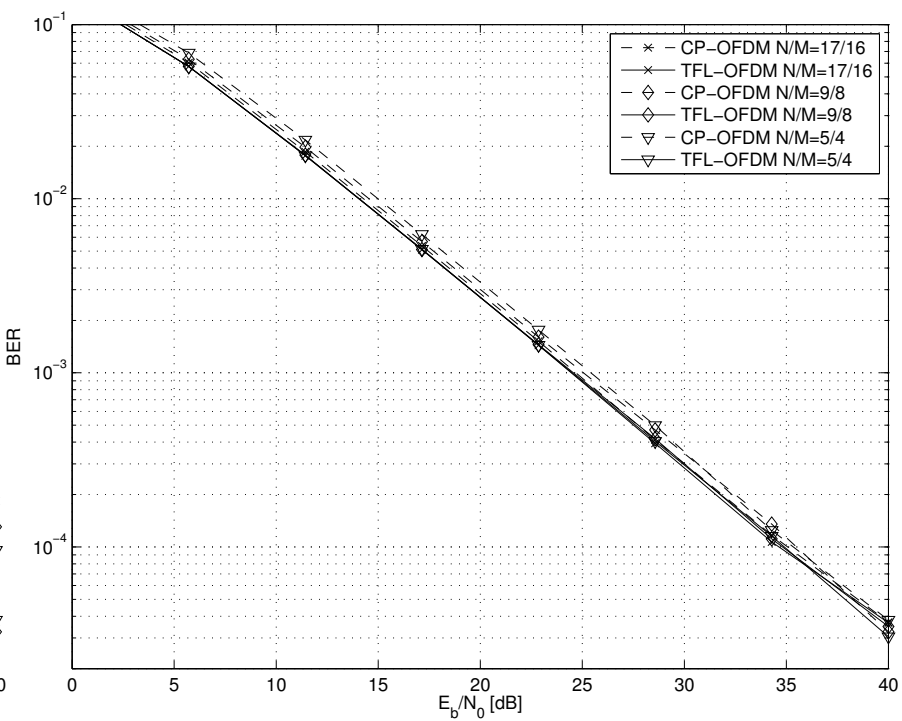

(b) BER performances of TFL-OFDM and CP-OFDM $\left(v_{\max }=3 \mathrm{~km} / \mathrm{h}\right)$.

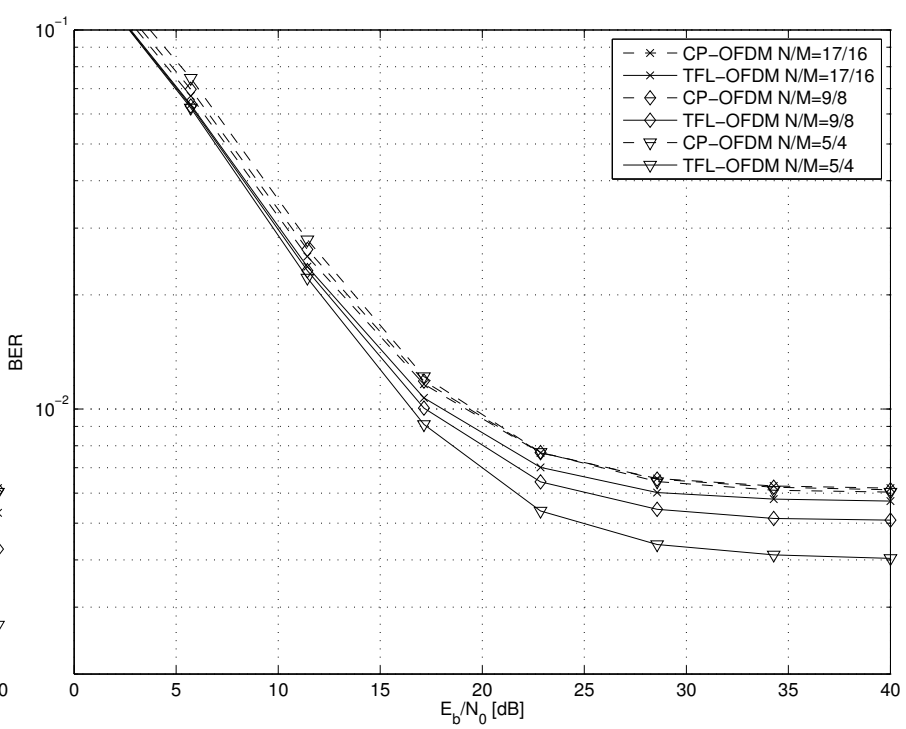

(d) BER performances of TFL-OFDM and CP-OFDM $\left(v_{\max }=350 \mathrm{~km} / \mathrm{h}\right)$.

Fig. 3: BER performances for pedestrian and vehicular scenarios in COST 207 TUx6 channel. Comparison between CP-OFDM and NR-OFDM for $M=4096$ and several values of $N / M$.

[8] P. Jung and G. Wunder, "WSSUS pulse design problem in multicarrier transmission," IEEE Trans. Commun., vol. 55, no. 9, p. 1822, 2007.

[9] F. D. Beaulieu and B. Champagne, "Design of prototype filters for perfect reconstruction DFT filter bank transceivers," Signal Processing, vol. 89 , no. 1 , pp. $87-98,2009$.

[10] D. Pinchon and P. Siohan, "Closed-form expressions of optimal short PR FMT prototype filters," in Proc. IEEE Global Telecommunications Conf. GLOBECOM '11, 2011.

[11] N. Moret and A. M. Tonello, "Design of orthogonal filtered multitone modulation systems and comparison among efficient realizations," EURASIP J. Adv. Signal Process, vol. 2010, pp. 10:1-10:11, January 2010.

[12] A. M. Tonello and F. Pecile, "Analytical results about the robustness of FMT modulation with several prototype pulses in time-frequency selective fading channels," IEEE Trans. Wireless Commun., vol. 7, no. 5, pp. 1634-1645, 2008.

[13] O. Christensen, Frames and bases: An introductory course. Birkhauser, 2008.
[14] P. Bello, "Characterization of randomly time-variant linear channels," IEEE Transactions on Communications Systems, vol. 11, no. 4, pp. 360393, 1963.

[15] R. Clarke, "A statistical theory of mobile-radio reception," Bell Syst. Tech. J, vol. 47, no. 6, pp. 957-1000, 1968.

[16] C. Iskander, "A MATLAB-based object-oriented approach to multipath fading channel simulation," Hi-Tek Multisystems, Tech. Rep., 2008.

[17] P. Jung, "Pulse shaping, localization and the approximate eigenstructure of LTV channels (special paper)," in Proc. IEEE Wireless Communications and Networking Conf. WCNC 2008, 2008, pp. 1114-1119.

[18] C. Siclet, "Application de la théorie des bancs de filtres à l'analyse et à la conception de modulations multiporteuses orthogonales et biorthogonales," Ph.D. dissertation, Université de Rennes 1, november 2002.

[19] L. C. Calvez and P. Vilbé, "On the uncertainty principle in discrete signals," IEEE Trans. Circuits Syst. II, vol. 39, no. 6, pp. 394-395, 1992.

[20] M. Failli, "COST 207 digital land mobile radio communications," Commission of the European Communities, p. 137, 1988. 